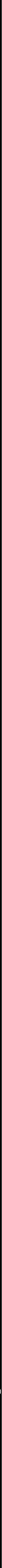




\section{Pensamiento de diseño, procesos creativos y literatura}

Diego Sheinbaum

En este estudio, el autor explora los puntos de confluencia entre el diseño y la literatura en lo que hace a sus procesos de creación. Con ello se pretende abonar al ámbito de la enseñanza, tanto del diseño como de la literatura, abrevando de conceptos torales en el ámbito del llamado Pensamiento de diseño (Design Thinking), como el de "salto creativo".

Palabras clave: literatura, procesos de creación, enseñanza, salto creativo, pensamiento de diseño. 


\section{Discusión}

El objetivo de este trabajo es explorar la manera en que los procesos creativos se dan en la literatura y el diseño. Mediante una revisión teórico-metodológica ilustrada con ejemplos de reconocidos profesionales se intentará demostrar que estas dos disciplinas pueden enriquecer su comprensión de la creatividad al iluminar sus procesos de manera mutua. De forma paralela se intenta mostrar que el diálogo entre ambas disciplinas puede contribuir al proceso de enseñanza-aprendizaje de la creatividad en las aulas; no sólo eso: los estudiantes y practicantes del diseño pueden obtener beneficios al explorar áreas importantes de las disciplinas literarias como son el arte de la narración, la retórica y la poesía; de la misma manera que los estudiantes y practicantes de la literatura pueden beneficiarse al conocer los procesos característicos del diseño, como es la elaboración de bocetos y prototipos, o al empamparse de la metodología que se ha desarrollado en el diseño para el trabajo colaborativo. Así, se busca continuar el diálogo, a veces explícito, la mayoría de la veces implícito, que se ha dado entre el diseño y la literatura. En esta área de intersección los estudios son escasos. Sin embargo, hay propuestas provocativas como la de Richard Buchanan, quien extiende el poder integrador del diseño en la sociedad contemporánea al establecer sus antecedentes en dos fuentes aristotélicas de los estudios literarios: La poética y La retórica. Así, la primera parte del ensayo se concentra en planteamientos generales sobre ambas disciplinas, mientras la segunda aborda el proceso creativo, en particular las etapas relacionadas con el pensamiento lateral o divergente, y de manera específica, explora los llamados "saltos creativos".

¿Qué entendemos por literatura y diseño? Ambos conceptos agrupan una pluralidad de prácticas, teorías, historias y críticas, cuyos significados han cambiado con el tiempo. Terry Eagleton (1996) enuncia tres definiciones alternativas de literatura —y las limitaciones de cada una de ellas - que pueden servir para comenzar el diálogo con el diseño. La primera entiende la literatura como escritura imaginativa, sinónimo de ficción. Para Eagleton el problema con esta definición es que deja fuera una buena parte de lo que consideramos textos literarios; por ejemplo, los ensayos de Montaigne o los sermones de Donne. Sin embargo, esta definición es útil si retomamos el sentido que tenía para Aristóteles. En su poética, el filósofo griego define el área de la ficción en contraposición con la disciplina de la historia. Mientras la historia se encarga de la verdad y lo sucedido, la ficción se encarga de lo probable. A la luz de esta definición, las reflexiones de Montaigne caben perfectamente en tanto reflexiones posibles.
Esta definición ampliada es útil por dos motivos. Primero, permite extender el área de la literatura a toda elaboración de historias ficticias; es decir abre el campo para estudiar expresiones cinematográficas, televisivas o de animación como fenómenos literarios, y con ello absuelve a la literatura de estar condenada a la exclusiva tecnología de la palabra escrita. En segundo lugar, Aristóteles está definiendo el área donde crecerá el diseño, por lo menos a partir de la Revolución industrial, el área de lo probable, muy importante como se verá más adelante (1995). La segunda definición de literatura es la que dan los formalistas rusos, quienes pasan por alto la dicotomía ficción-realidad y se concentran en el uso del lenguaje. Para ellos la literatura involucra el uso singular de la lengua. La literatura es todo aquello que transforma e intensifica el lenguaje ordinario, lo que se desvía sistemáticamente del habla cotidiana. Por eso los formalistas se concentran en las características internas del texto. Eagleton señala que el problema con esta definición es que no existe un lenguaje ordinario en sí mismo. Todo depende del lugar, el tiempo, la clase social y el grupo étnico donde uno se encuentre. Lo que para algunos es normal, para otros es extraño. A pesar de esta limitación, los estudios formalistas y estructuralistas refinaron la forma en que entendemos los textos literarios en tanto artefactos y con ello, como se verá más adelante, nos dieron nociones que pueden iluminar los procesos de diseño. Por último, Eagleton propone que la literatura es toda escritura significativa, cualquier texto que una persona considere valioso. En este sentido, lo que crea la literatura no es tanto una característica inherente al texto sino la manera en que nos aproximamos a él, nuestra actitud de reverencia (1996, p. 1-14). Esta última definición es útil porque desenmascara los valores y las ideologías que están detrás de aparentes juicios objetivos. Aunque este trabajo fija su atención en las consecuencias de las primeras dos definiciones, es importante tener en cuenta las instituciones, valores e ideologías que están detrás de los argumentos que definen lo qué es y lo qué no es literatura, lo qué es y lo qué no es diseño.

De manera similar a la noción de literatura, el diseño es un concepto abierto y flexible que, en la medida que abarca más fenómenos, pierde su sentido (Lindberg et al. 2010, 32). En doscientos cincuenta años de historia, el diseño pasó de ser una actividad comercial, a ser una profesión segmentada, a volverse un campo de investigación, hasta convertirse en una potencial arte liberal, o digna de ser parte de la formación general que reciben todos los alumnos (Buchanan. 1995b, p. 3). Esta última revolución aparece asociada a lo que se ha denominado Design Thinking, término que se puso en boga en las últimas décadas del siglo XX y que ha vivido una serie de evoluciones. En un principio era una noción descriptiva para explicar la ma- 
nera en que los diseñadores razonaban a la hora de practicar su oficio; después se convirtió en un término normativo que daba cuenta de las etapas y las formas que debía tener el proceso creativo para ser exitoso; por último, dejó las fronteras del diseño para volverse una forma de pensamiento tan importante como el razonamiento deductivo o inductivo; una forma de enfrentar problemas y encontrar soluciones que podía aplicarse a las más distintas áreas. A esta última evolución contribuyó el éxito que tuvieron empresas de diseño como IDEO dentro de grandes trasnacionales. En el contexto de la economía globalizada, Design Thinking se volvió sinónimo de innovación y, por lo tanto, de sobrevivencia. De manera paralela, en el mundo de la educación, el diseño se convirtió en una fuerza integradora, una forma de articular la fragmentación de las disciplinas. Escuelas como The Rotman School of Managment en Toronto, La d. desing en Stanford y Centro de diseño,cine y televisión en la Ciudad de México, se abocaron a impartir una educación universitaria que contemplaba al diseño como la columna vertebral de una gama amplia de disciplinas (Lindberg. 2010, p. 31-36).

Esta tendencia integradora es tan frecuente hoy en el diseño como lo fue en la literatura durante los siglos XIX y XX. Entonces, la literatura, en opinión de sus principales promotores - Herder, Goethe y Vico- era un medio para conocer a otras culturas y apreciarlas en sus peculiaridades; la literatura mundial (Weltliteratur) era una forma de civilizar a través del conocimiento, de buscar el entendimiento y el diálogo entre grupos humanos (Auerbach. 2009, p. 125-138). Fue en este momento que la filología se convirtió en el corazón de las humanidades y éstas en la formación indispensable de todo estudiante: el arte liberal que se enseñaba a todos los alumnos, es decir esa educación que se concentra en la libertad y que logra hacer sentido de las más diversas experiencias del mundo, un disciplina que intenta darle a sus estudiantes herramientas para enfrentar los problemas de la vida.

Curiosamente Richard Buchanan formula la concepción del diseño en tanto arte liberal de una sociedad tecnológica. En sus estudios ubica los orígenes del talante integrador del diseño en las primeras décadas del siglo XX, alrededor de Walter Gropius y de la escuela de Bauhaus. Gropius se propuso formar una generación de artistas que dejarán las alturas del Olimpo para regresar a solucionar los problemas y las necesidades de la gente. Es decir, se propuso volver al arte útil. De esta manera rompió el cerco que los artistas habían trazado alrededor de la exploración formal y fomentó pesquisas simultáneas de la formas y de las funciones, del deseo y las necesidades. Así intentó vincular el arte a los procesos productivos y apostó por liberar al hombre de la máquina, pero sin despojarlo de las ventajas que le ofrecían las nuevas tecnologías, o dirigiéndolas

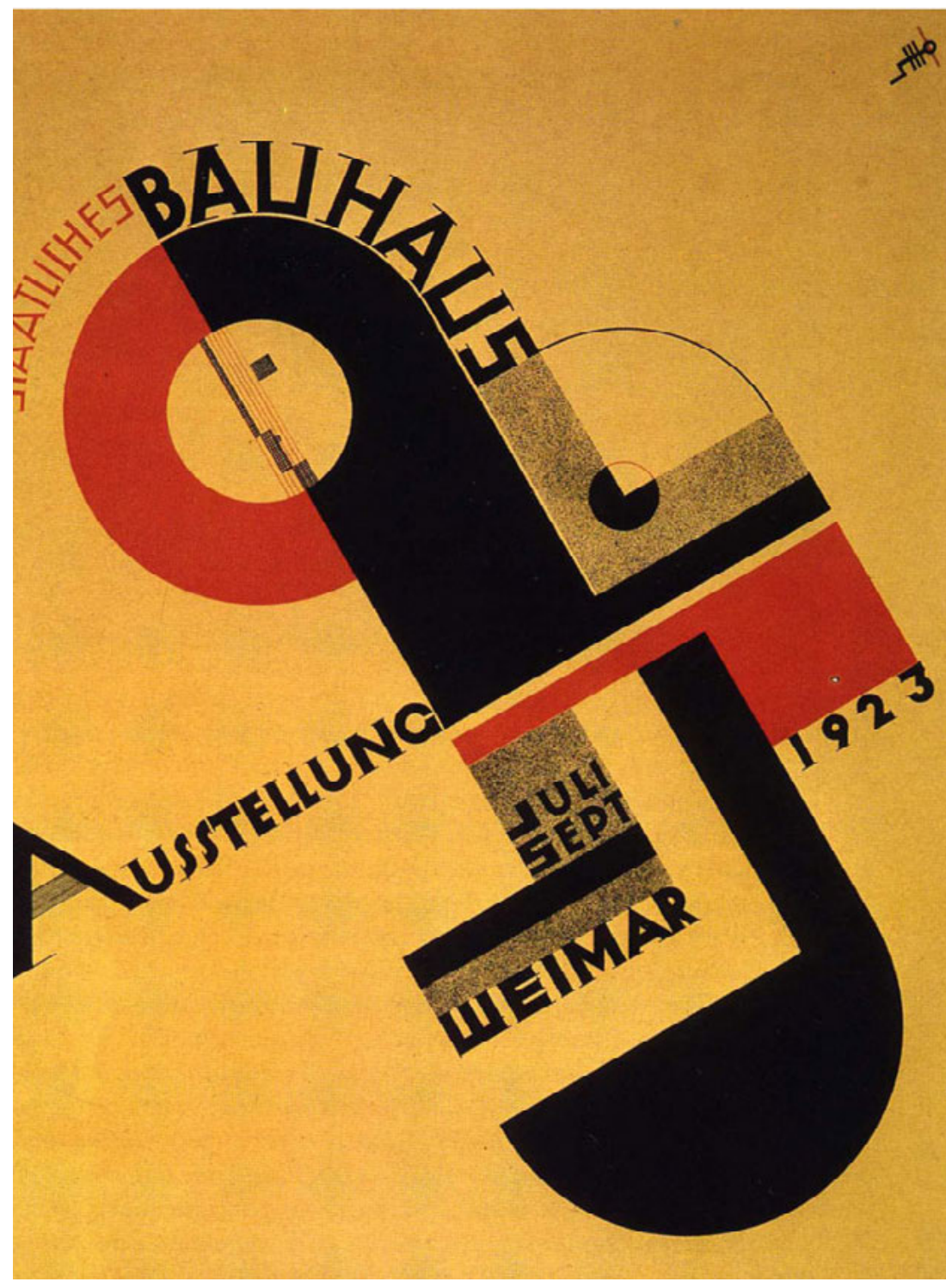

http://forums.watchuseek.com/

a fines humanos. Bajo su impulso el diseño se convirtió en el área de confluencia de la ciencia, la tecnología y el arte, el cual buscaba superar los obstáculos para satisfacer las necesidades y deseos del individuo, las organizaciones y las sociedades. A Gropius le preocupaba tanto la tendencia del arte por el arte como la exclusiva mentalidad materialista que consideraba el negocio un fin en sí mismo (Buchanan. 1995a, p. 30-36).

Buchanan agrega dos elementos de carácter epistemológico para fundamentar el roll protagónico del diseño en las prácticas y saberes de la sociedad contemporánea. El primero es el principio de incertidumbre, desarrollado por John Dewey. Para el filósofo norteamericano los procesos cognitivos del hombre contemporáneo ya no se dan entre una mente con su equipo de poderes completos y una materia completa en sí misma. Ahora estos procesos ocurren en una interacción indefinida que toma lugar dentro de una naturaleza que no está fija ni completa, sino que es capaz de ser dirigida en nuevas direcciones y a diferentes resultados, a través de operaciones in- 
tencionales (Dewey, J. 1960, p. 290-291). Así, la capacidad de inyectar intencionalidad a la naturaleza es lo que convierte al diseño en una disciplina indispensable en el contexto contemporáneo. El segundo elemento se enfoca en el tipo de problemas que enfrenta el diseñador. En 1971 Horst Rittel los definió como "problemas malvados" porque, a diferencia de los problemas en la ciencia, no tienen una solución única ni siquiera pueden plantearse de manera unívoca, sino que aceptan varias configuraciones. Esto hace que los problemas del diseño sean semejantes a los problemas cotidianos, donde la información no es completa y hay un ingrediente muy importante de indefinición en cuanto al área problemática.

Estas razones explican la importancia del diseño dentro de una educación general. Sin embargo, el movimiento más radical de Buchanan nos lleva hacia el pasado, nos lleva de regreso a la literatura, a dos vetas clásicas de esta disciplina: la retórica y la poética. Siguiendo a Herbert Simon, Buchanan ve en La poética de Aristóteles el origen de una ciencia de lo artificial, cuyo terreno no es lo necesario, sino lo posible. Aunque Aristóteles sólo se ocupa del arte verbal y, en particular, de la tragedia, ahí están los fundamentos para estudiar las artes productivas y reproductivas, las fabricaciones de la imaginación. Pero es, sobre todo, en la retórica donde Buchanan encuentra el impulso integrador que puede articular las más diversas prácticas del diseño. En la tradición clásica la retórica es la ciencia de la persuasión, el arte de descubrir argumentos lógicos, éticos y emocionales para tratar de generar creencia e identificación ya sea sobre el pasado, el presente o el futuro (Buchanan, R. 1989, p. 93). Buchanan enfatiza que la revolución tecnológica descubrió una nueva dimensión de la retórica: el poder de los objetos para provocar efectos similares. A través de moldear nuevos productos, los diseñadores influencian directamente las acciones de los individuos y de las comunidades, cambiando actitudes y valores, transformando a la sociedad de manera completa. Desde esta perspectiva, la revolución tecnológica logró algo que parecía imposible: zanjar la distancia entre las palabras y los objetos. Lo que antes la retórica lograba con las palabras -informar, instruir y complacer- es hoy conseguido a través de la manera en que el diseño participa en todas las áreas de la producción. A este universo Buchanan lo divide en cuatro vastas áreas. La primera es el diseño de la comunicación, que agrupa al diseño gráfico, a la mercadotecnia y a la publicidad, pero también a la fotografía, el cine y la televisión. En esta área se exploran los problemas en la comunicación de datos, ideas y argumentos a través de la síntesis de imágenes y palabras. La segunda área corresponde al diseño de objetos, que se ocupa de la forma y apariencia de objetos cotidianos: la ropa, objetos domésticos, herramientas, maquinaria, vehículos y una lista interminable. La tercera área se enfoca en el diseño de acciones, en la organización de estructuras y estrategias de servicios. Por último, la cuarta área se encarga de entornos, sistemas complejos y hábitats, que van desde los sistemas de computación hasta el urbanismo. Buchanan afirma que en los productos de estas cuatro áreas se encarnan argumentos para convencer a los usuarios de que su vida mejorará Buchanan (1989, p.10). Incluso emparenta el proceso creativo con las etapas de la retórica: el descubrimiento, invención, argumentación y planeación. La persuasión y deliberación, propias de la retórica, se dan en la manera en que el diseñador incorpora y moldea los argumentos del cliente, de los especialistas técnicos, de otros diseñadores y de los futuros usuarios. Tim Brown retoma esta idea cuando sugiere que el diseñador tiene que balancear la perspectiva de los usuarios, de la tecnología y de los negocios, aunque, para él, el acento siempre está en los usuarios. Por ello lo diseñadores deben observar cómo la gente se comporta, cómo el contexto afecta su reacción a productos y servicios; deben tomar en cuenta el significado emocional de las cosas y la manera en que funcionan. Su punto de partida es identificar las necesidades latentes — no dichas - de la gente y transformarlas en oportunidades Brown (2009, p. 229).

La argumentación de los diseñadores se encarna en la serie de planes, propuestas, bocetos, modelos y prototipos que son presentados para comprender, comunicar y producir. El problema estriba, para Buchanan, en que este modo de comunicación no ha sido comprendido cabalmente. Y la forma de humanizar al diseño consiste, para él, en verlo bajo esta luz. Porque la retórica abre la posibilidad de controlar y hacer racional las explosiones de diseño, abre los ojos del diseñador y del usuario para que perciban la argumentación detrás de cada producto. Por eso sería importante que los estudiantes de diseño exploraran nociones del viejo arte de la persuasión. ${ }^{1}$

Una propuesta cercana a la de Buchanan es la que ha formulado Nigel Cross (2006, p. 1-13). La forma en que este autor entra al debate hace referencia también a la literatura, en particular al famoso ensayo de C.P. Snow, The Two Cultures, donde el crítico literario plantea que la ciencia y las humanidades se han convertido en dos áreas autónomas sin ningún tipo de diálogo Snow (1964). Para Cross estas dos áreas han dominado los sistemas sociales, culturales y, sobre todo, educativos. Sin embargo, cree que el diseño se puede convertir en una tercera área, tan importante como las otras en la formación general de los alumnos. Para comprobarlo, Cross muestra que

1 El planteamiento de Buchanan ha encontrado eco pero también una serie de críticas. Quizá una de las más interesantes es la que se apoya en la argumentación del mismo Aristóteles para criticar el movimiento ético del diseño de los últimos años. (Wang, 2013). 
el diseño tiene su propio objeto de conocimiento, su propia metodología y su propio lenguaje. Si la ciencia se encarga de estudiar lo natural, y las humanidades la experiencia humana, el diseño se encarga de lo artificial. Si la ciencia utiliza el lenguaje de los números, y las humanidades utilizan las palabras, el diseño despliega un importante lenguaje visual alrededor del modelaje. Esta forma particular de pensar se vuelve más notoria en la comparación directa con la ciencia. Mientras que ésta busca conocer la ley detrás de los problemas, el diseño se enfoca en conocer la solución. Si la ciencia es por naturaleza analítica, el diseño es constructivo; si la ciencia se encarga de cómo son las cosas, el diseño de cómo podrían ser. Cross caracteriza la forma particular de pensar del diseño a través de tres aspectos: 1) enfrenta problemas "mal definidos" o "malvados", 2) Construye la solución del problema al mismo tiempo que lo delimita, 3) utiliza códigos no verbales que traducen los requerimientos abstractos en objetos concretos.

Comparada con la propuesta de Richard Buchanan, la de Nigel Cross parece ser una propuesta menos grandilocuente; no intenta erigirse sobre las otras disciplinas y articular sus saberes, sino que busca reconocer un tipo de pensamiento que es común a todas las prácticas del diseño; es decir, intenta construir los fundamentos de un diseño con " $d$ " mayúscula. Una de las partes más interesantes de los estudios de Cross son los análisis de las prácticas comunes a todos los diseñadores, la exploración que hace de sus habilidades y la forma en que pueden ser desarrolladas. En esta área el diálogo con la literatura se vuelve más interesante. ${ }^{2}$ Estos procesos se caracterizan porque el problema y la solución se construyen de manera simultánea. En vez del proceso analítico de la ciencia donde, primero, se descompone el problema en sus distintos elementos para, después, buscar una solución, en el diseño los sub-problemas sólo aparecen con la exploración de las sub-soluciones. Esta simultaneidad no ha impedido que los diseñadores distingan varias etapas en su proceso de creación. A grandes rasgos se puede hablar de tres fases o espacios que se entremezclan: 1) La iluminación del terreno del problema 2) La iluminación del terreno de la solución y 3) La encarnación de soluciones.

Para Tim Brown, en estas etapas interactúan dos tipos de razonamiento, lo que llama pensamiento divergente y pensamiento convergente. Este diseñador imagina el proceso creativo bajo la forma de un cono, donde en las primeras etapas prevalece la divergencia y, conforme nos acercamos al final, la convergencia

2 Otra interesante forma de entender la función integradora del diseño es la que proponen en su artículo Lindberg y sus colaboradores. Para ellos el diseño, más que una nueva retórica de la sociedad contemporánea, más que un área de conocimiento autónoma, ofrece una metodología para el trabajo creativo colaborativo. Si bien la intención de fondo sigue siendo la misma, contrarrestar la fragmentación de los saberes, la propuesta de estos diseñadores es aún más concreta, incluso llegan a formular siete etapas en el proceso creativo (Lindberg, 2010, p.31-36). se impone para alcanzar soluciones (Brown, 2009, p. 66-70). Jonathan Edelman (2012) y un grupo de colaboradores han representado este proceso dual a través de las prácticas para cazar caribúes que sigue los orochons. Esta actividad logra sus resultados gracias a la clara distinción de dos roles: los cazadores y los recolectores. Las trayectorias enrevesadas de los primeros son parecidas a las vísceras. Como si estuvieran en un sueño, los cazadores van armando y desarmando pistas, cambian de dirección conforme responden a las mínimas estimulaciones del paisaje, a lo que sus sentidos detectan: el movimiento de las hojas, los rastros sobre la nieve, los diferentes silencios. Una vez que cazan a su presa, la dejan en ese sitio y siguen con su deambular para hacerse de nuevas víctimas. Entonces entran los recolectores en escena. Viajan en trineos para recoger la caza y llevarla de regreso a la aldea. A ellos ningún estímulo los aleja de la línea recta: la forma óptima de recolección.

Curiosamente, Peter Brooks (1992) representa la forma que toman las historias en la ficción como un arabesco, una figura muy cercana a la víscera. Brooks señala que todo avance en la ficción supone una constante desviación y encuentra en Tristam Shandy un ejemplo paradigmático. En esta novela el afán del narrador de contar su historia siempre es diferido por las digresiones que lo alejan de su objetivo. De manera más concreta, el famoso profesor de escritura creativa de la Universidad de Columbia, Stephen Koch, traza una distinción entre los conceptos de historia y trama que parece acercarse mucho a la idea de cazadores y recolectores planteada por Edelman. La historia para Koch tiene que ver con el sentido intuitivo en que desarrollamos una serie de sucesos y personajes; es decir, la manera en que deambulamos de manera casi ciega. Mientras que la trama es una operación más cercana al cálculo, a la manera en que optimizamos nuestros descubrimientos. Uno de los problemas más frecuentes que encuentra Koch entre sus alumnos es que quieren darle forma a la historia sin haber explorado lo suficiente, quieren recolectar cuando apenas han capturado una presa (Koch, 2003).

En el área del diseño, Bruce Mau lleva toda una carrera preguntándose si es posible enseñar a los estudiantes a dar rienda suelta a estos procesos de divergencia. A través de sus experiencias ha llegado a la conclusión que es importante aprovechar la ventaja que nos da la ignorancia. Por eso recomienda a sus alumnos y colaboradores dos cosas. Primero: hacer preguntas estúpidas sobre los problemas. Segundo: perderse en el bosque. En ambos casos se trata de convertir la falta de conocimiento y certidumbre en un incentivo a la imaginación; aprovechar ese momento en que la especulación puede volar sin las amarras de las convenciones, sin las certidumbres de las especialidades. Por la misma razón, Tim Brown y George Kembel 


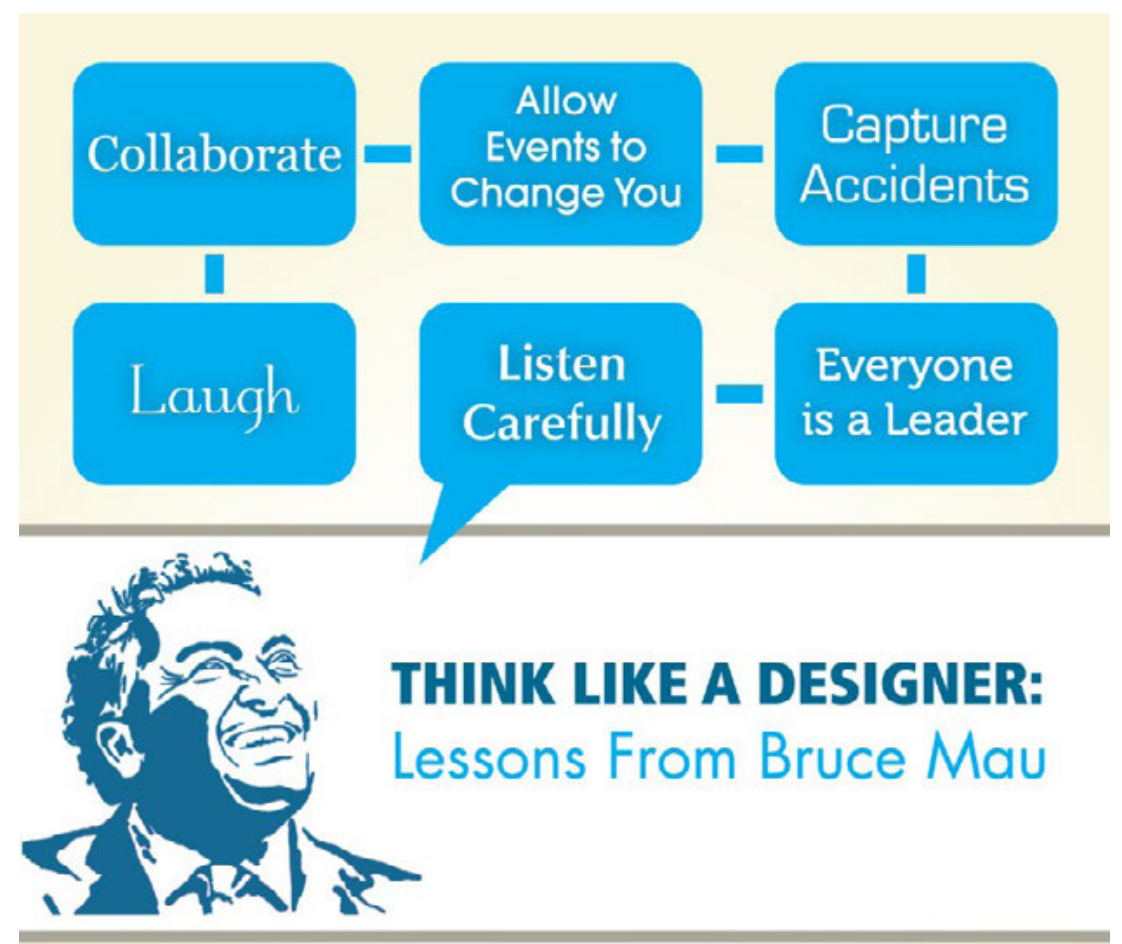

Bruce Mau is a renowned designer that has influenced
projects as far reaching as the look of Indigo Books, to
the Walt Disney Concert Hall in Los Angeles. The rules
above are from his Incomplete Manifesto for Growth.
"Creativity has been made into a mythology. Certain
people have it and certain people don't. The reality
is it's hard work."

http://tribehr.com

recomiendan adoptar el punto de vista de los usuarios, de personas no expertas. Incluso Mau sugiere buscar los casos extremos: usuarios sumamente torpes o sumamente sofisticados (Berger, 2010, p. 53). Sin embargo, la idea de hacer preguntas estúpidas es demasiado simplista. Sobre todo si consideramos, con Tim Brown, que hacer las preguntas adecuadas a menudo determina el éxito de un nuevo producto o servicio ${ }^{3}$. De esta manera, pareciera que incluso detrás de la invitación a hacer preguntas estúpidas hay un arte, para el cual es tan importante recuperar el famoso por qué de los niños, como estar conscientes del proceso de una manera que los niños no lo están, estar conscientes de que hacer ciertas preguntas nos ofrece la oportunidad de no dar por hecho nada, de ir más allá de las convenciones que nos determinan; la oportunidad de re-encuadrar el problema, redefinir sus restricciones y abrir el camino para una respuesta más innovadora. ${ }^{4}$

Los consejos de Bruce Mau persiguen la meta de enfrentar los problemas y las situaciones de una manera fresca, artilugio que ha sido una de las especialidades de la literatura. A través del punto de vista de niños, locos y tontos la literatura nos ha logrado mostrar aspectos inesperados de la realidad y de las convenciones sociales. Cervantes renueva la visión de España a través de su enloquecido Quijote. Algo similar hará Dostoievski con la sociedad rusa a través de El idiota. El punto de vista de estos personajes nos permite no dar por hecho las convenciones bajo las cuales opera el orden social y, por lo mismo, nos permiten ir más allá de la costumbre y del automatismo. Ejemplos más recientes podemos encontrarlos en las novelas del escritor sudafricano J.M. Coetzee. En Live and Times of Michael $K$ (1983) el autor nos da una extraña visión del Apartheid — tan abordado por las letras sudafricanasdesde la perspectiva de un hombre con labio leporino, que ha vivido entre orfanatorios y parques públicos, y que en vez de una racionalidad ha desarrollado la sensibilidad de un animal. Mientras que en su siguiente novela, Foe (1986), Coetzee nos arroja junto con Susan Barton a la conocida isla de Defoe para darnos el punto de vista de una mujer de esa vida que llevaban Crusoe y Viernes. Aunque los eventos son los mismos que en la célebre novela, la historia de la isla aparecerá bajo una luz insospechada, gracias al punto de vista de Susan.

Esta tendencia de la literatura ha sido extrapolada por los formalistas rusos, quienes han visto el gran valor de la literatura en la des-familiarización o extrañamiento. Para ellos la literatura combate los peligros del hábito. Lo habitual, nos dice Shklovsky, devora la ropa, los muebles, a las esposas, incluso a la guerra. El hábito nos vuelve inmunes a la singularidad de los objetos y los sucesos. Por el contrario, la literatura nos permite recuperar la sensación de vida, nos hace sentir las cosas, percibir a la roca rocosa. Así, el fin del arte es dar la sensación del objeto como visto, no como reconocido. La técnica de la literatura es hacer las cosas no-familiares, hacer las formas oscuras, de tal manera que se extienda la percepción (Shklovsky, 1990, p. 5). Este impulso central en la literatura corre en la misma dirección que la recomendación que hace Tim Brown de abrir los ojos: "Entre más familiar la situación, más la damos por hecho. Los buenos diseñadores observan, pero los mejores observan lo ordinario." (Brown, 2009, p. 237). En este mismo sentido, uno de los teóricos más importantes del género de la novela, Mijail Batjín, ha señalado un importante antecedente de las novelas de Cervantes y Dostoievski en los diálogos platónicos. Sócrates es el paradigma de ciudadano que hace preguntas estúpidas, que no acepta respuestas con-

3 “¿Cubre las necesidades de la gente a la cual está dirigida? ¿Aumenta su significado, así como su valor? ¿Inspira un nuevo comportamiento que, para siempre, estará asociada con él? (Brown, 2009, p. 230)

4"En vez de aceptar una restricción dada, preguntarse incluso si este es el verdadero problema a resolver ¿Qué necesitamos autos más rápidos o un mejor transporte? Hacer estas preguntas, en el largo plazo, aumentará las posibilidades de gastar la energía en los problemas más adecuados" (2009 p. 237). 
vencionales, que nos recuerda el difícil arte de desnudar el conocimiento para formular lo elemental. A esto hay que añadir la energía psíquica que la ignorancia libera, porque cuando uno "se pierde en el bosque" en palabras de Mau: "todo lo que te rodea adquiere una importancia añadida. De pronto, tienes que navegar y negociar cada detalle del entorno, procesándolo mientras tratas de orientarte. Cuando la gente está muy alerta es un tiempo ideal para especular y experimentar."( Berger, 2010, p. 53).

Esta incitación a pensar de una forma distinta a la convencional ha sido reformulada por Edward De Bono mediante el concepto de "pensamiento lateral", en contraste con el pensamiento lógico, al cual denomina vertical. Si la función del pensamiento lógico es el inicio y desarrollo de modelos de conceptos, el pensamiento lateral se encarga de la reestructuración de estos modelos y la creación de otros. Si el pensamiento vertical profundiza una ruta, el pensamiento lateral se encarga de abrir nuevos caminos (de Bono, 1970, p 15-17). Para De Bono el gran problema del pensamiento vertical es que avanza por un camino sólo a través de la exclusión de otras bifurcaciones, mientras el pensamiento lateral no selecciona una ruta, sino que intenta seguir varias (1970, p. 47) Una de las implicaciones más importantes de dicha distinción es que mientas el pensamiento vertical se basa en la secuencia de ideas, el lateral puede efectuar saltos. Esta habilidad es fundamental para el proceso de creación de historias y para la elaboración de diseños, ya que a menudo la manera en que abordamos un problema o lo primeros descubrimientos en una historia determinan el resto del proceso, y sólo a través de saltar fuera de la ruta podemos evitar los callejones sin salida. Este es el principal problema que Koch señalaba entre sus estudiantes, la tendencia a quedarse encerrados en una situación, sin buscar más elementos por otras rutas. En el mismo sentido, Nigel Cross señala la importancia de buscar más de una solución para un problema de diseño, porque, a menudo, una sola solución nos da una perspectiva muy parcial y tendenciosa. Explorar por lo menos dos soluciones permite alcanzar, a través de la comparación de los sub-problemas, una mejor perspectiva de la naturaleza del problema. ${ }^{5}$ En el mismo sentido, Roger Martin argumenta que los pensadores que explotan ideas contrarias u opuestas para construir nuevas soluciones tienen ventaja sobre pensadores que sólo consideran un modelo a cada tiempo. A esta actitud la denomina pensamiento integrador y se caracteriza por resistirse a la disyuntiva esto/o aquello y preferir "ambos/y". Es decir es un pensamiento que ve las relaciones no lineales y multidireccionales como una fuente de inspiración y no de contradicción (Martin, 2006).

5 No sólo eso, nos dice Cross, a través de soluciones provisionales descubrimos aspectos relevantes del problema. (Cross, 2006, p. 18)
Linda Hutcheon ha identificado este tipo de pensamiento en algunos de los más grandes exponentes de la literatura posmoderna. Ha descubierto en escritores tan disímiles como Tony Morrison, W. G. Sebald, Graham Swift y J.M. Coetzee esta forma de habitar las paradojas, de combinar el impulso auto-referencial y paródico del modernismo con un impulso contrario que recupera el valor de la narración, de las convenciones y de rescatar el contexto histórico, social y político. Su propia definición del posmodernismo opera bajo esta base. Para ella la definición popular del posmodernismo caracterizado por discontinuidades, disrupciones, dislocaciones, des-totalización e indeterminación, sólo es la mitad del fenómeno, y hay que describir al postmodernismo como un fenómeno contradictorio: uno que usa y abusa, instala y subvierte los conceptos tradicionales de la literatura. Para Hutcheon la etiqueta "post" significa, en referencia a estos destacados escritores, una relación contradictoria, de dependencia e independencia con el modernismo que los precedió. No es "un simple rompimiento con el modernismo, ni tampoco una automática continuidad. Es ambos y ninguna."( Hutcheaon, 1988).

Así, el pensamiento lateral permite mantener abierta la posibilidad de reestructuración; es decir, de cambiar el orden y la importancia con que aparecen los elementos. Esta es la principal experiencia que enseña Uzyel Karp en la clase de "proceso creativo" que imparte en Centro de diseño, cine y televisión. De manera explícita, Karp ejercita la capacidad de divergencia en sus alumnos a través de dos ejercicios. En el primero, grupos de tres alumnos tiene que recolectar veinte piedras y clasificarlas de acuerdo a las más variadas características que encuentren en ellas. En un segundo ejercicio, a cada alumno se les asigna un objeto sencillo, como un llavero o un bolsillo, y se les pide que los exploren a través de variaciones visuales. Ambos ejercicios logran romper con las formas convencionales de organizar el material. Ambos invitan a los estudiantes a aventurarse en la oscuridad.

En el área de los estudios literarios, la formulación del pensamiento lateral encuentra su contraparte en la importante teoría de Roman Jakobson sobre las dos operaciones principales a través de las cuales construimos cualquier lenguaje ( Jakobson, 1995). En su famoso artículo, "Dos aspectos del lenguaje y dos tipos de desórdenes afásicos", Jakobson distingue entre las operaciones sintagmáticas y paradigmáticas. Las primeras ocurren en el eje de combinación del lenguaje, mientras las segundas en el eje de selección. Jakobson explica que cuando construimos palabras, frases $\mathrm{u}$ oraciones siempre trabajamos a través de la selección entre posibilidades y la combinación de los elementos elegidos. Estas dos operaciones constituyen dos polos del lenguaje que Jakobson denomina metonímico y metafórico. Así en la literatura 
la prosa normalmente gravita hacia el polo metonímico, hacia la construcción a través de elementos contiguos o unidos por la causalidad; mientras que la poesía gravita hacia el polo metafórico, donde, más que la construcción lineal, lo que opera es un sistema de equivalencias, de grados de semejanza y diferencia, tanto en el tamaño de las palabras, como en sus sonidos y sentidos. Así, una forma alternativa de desarrollar la capacidad de pensar simultáneamente, es decir lateralmente, sería hacer conscientes a los alumnos del polo de selección que muchas veces opera en ellos inconsciente y automáticamente; hacerlos conscientes de las relaciones no sólo lineales sino simultáneas que se dan entre distintos elementos. La poesía es un excelente vehículo para ello porque, al mismo tiempo que su sentido se construye linealmente, establece relaciones por encima del flujo temporal y lineal de la sintaxis; es decir, la poesía ejercita la capacidad para percibir dentro de los problemas nuevas configuraciones. Para mostrar cómo sucede esto en la práctica vale la pena detenerse en uno de los poetas con mayor habilidad para ir más allá del pensamiento lineal. Rilke en sus poemas es capaz de renovar nuestra concepción incluso de la rosa, una de las figuras más convencionales y tratadas en la poesía lírica. En los pétalos de la rosa Rilke ve párpados que, en vez de abrirse, se cierran al deseo: "Rose, o pure contradiction, desire to be no one's sleep beneath so many lids. ${ }^{6} \mathrm{O}$, percibe en sus formas arremolinadas el enojo de dos muchachos peleándose mientras ruedan por una ladera, actores mentirosos y caballos desbocados cuyos ojos y dientes se pelan como si fueran cáscaras:

\footnotetext{
You've seen their anger flare, seen two boys

bunch themselves into a ball of animosity

and roll across the ground

like some dumb animal set upon by bees,

you've seen those carny barkers, mile-high liars,

the careening tangle of bolting horses,

their upturned eyes and flashing teeth,

as if the skull were peeled back from the mouth.?
}

Esta capacidad paradigmática para encontrar patrones y sentidos ocultos, a menudo ha sido llamada inspiración o "repentina iluminación" por los poetas. Los diseñadores no se han quedado atrás en acuñar términos para este fenómeno. Bruce Mau llama a dichos actos "saltar la barda", mientras que Nigel Cross los ve más como una manera de "tender puentes" entre el área del problema y el área de solución. Quizá la distinción más apropiada es la que hacen Edelman y sus colaboradores al distinguir entre diseño gradual y diseño radical. Esta distinción tiene la ventaja de centrarse en los fenómenos de re-diseño para entender los saltos radicales, ya que estas actividades permiten evaluar las diferencias con el modelo que ha servido como punto de partida (Edelman, 2012).

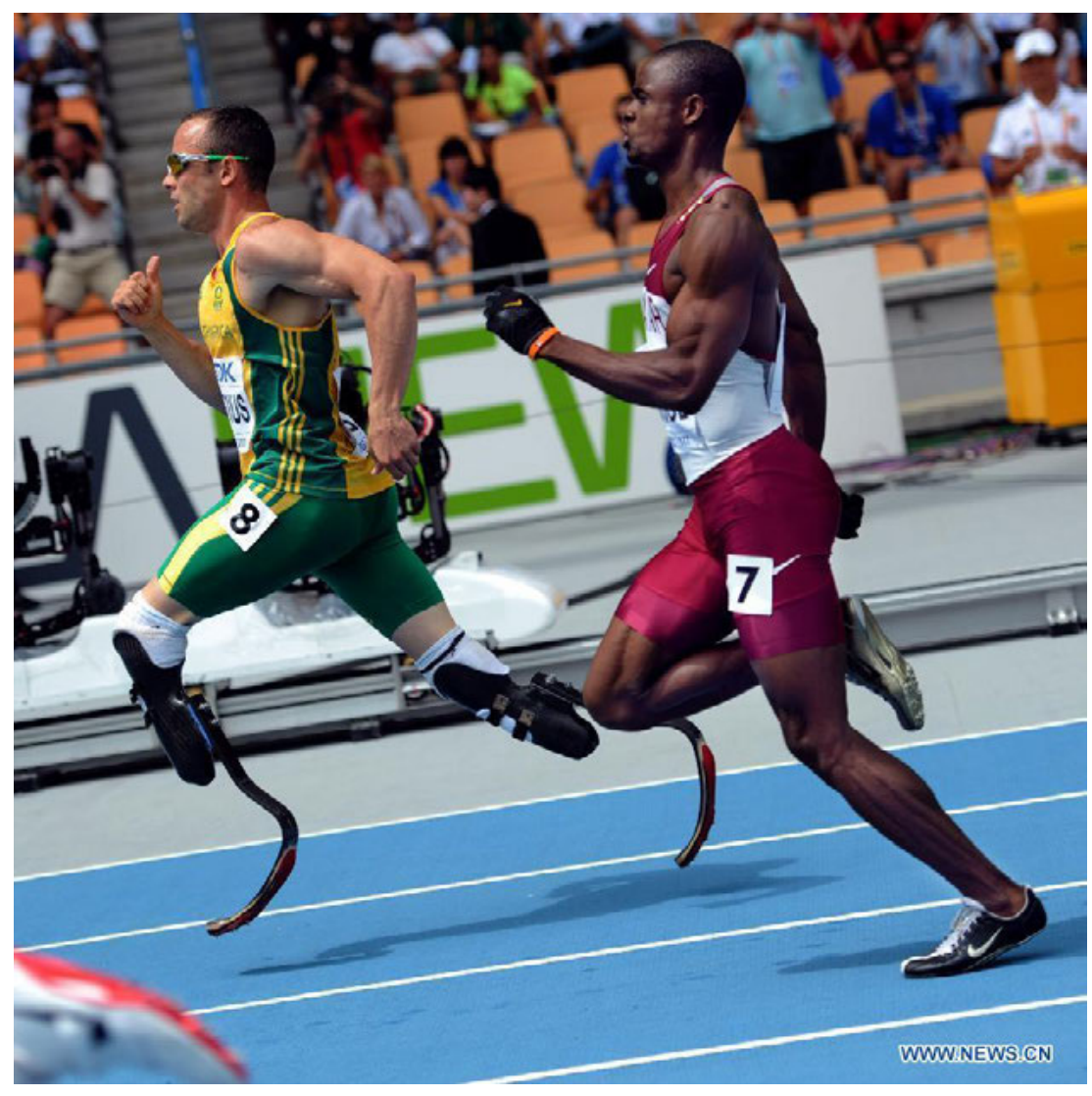

Oscar Pistorius

Más allá del nombre, uno de los ejemplos más famosos de "saltar la barda" en diseño es el invento de Van Phillips de la prótesis "el pie flexible de chita," el cual se volvió celebre en las competencias olímpicas cuando el atleta Oscar Pistorius utilizó las prótesis para competir con los hombres más veloces del mundo en la carrera de cien metros. En su libro Warren Berger recupera el proceso creativo que llevó a Van Phillips a su innovador diseño. Víctima de un accidente de esquí acuático, Phillips había perdido su pierna izquierda y durante los siguientes años se obsesionó con la idea de diseñar un repuesto. Para ello creó cientos de prototipos, horneándolos en su casa, luego probándolos él mismo, hasta que su modelo nació de una inesperada combinación. El padre de Phillips tenía una espada samurái con forma de "C" y Phillips siempre había estado impresionado por la flexibilidad de su hoja. Al mismo tiempo, Phillips estaba estudiando la mecánica de los trampolines de clavados y se preguntaba si el mismo tipo de fuerza se podía usar para lo prostético. En medio de esto se enteró cómo funcionaban las patas trase-

6 Las traducciones y notas a los poemas de Rilke son de Gass, W. H. (2000, pp. 3-5). Lamentablemente, las traducciones en español no le hace justicia al texto original: "Rosa, oh contradicción pura, alegría/ De no ser sueño de nadie bajo tantos párpados."

7 (Ibid., p. 4.) "Coléricos, has visto llamear, has visto a dos muchachos/ apelotonándose en una sola cosa, /que era odio y se revolcaba por el suelo/como un animal agredido por abejas;/ actores, fanfarrones amontonados, caballos furibundos que se desploman/arrojando la mirada, enseñando la dentadura, /como si el cráneo saliera, pelado, de la boca." (Rilke, 1991, p. 209) 
ras de la chita, con los tendones comprimiéndose y liberándose de una manera que brindaba gran elasticidad. Phillips comenzó a relacionar todo esto para intentar saltar la barda hacia una mejor pierna artificial. Su invento cambió la industria de las prótesis, así como la propia vida de Phillips, permitiéndole correr e involucrarse en otros deportes (Berger, 2010, p. 48-49).

Nigel Cross elabora un estudio mucho más detallado del proceso creativo en torno al Protocolo del Taller de Diseño de Deft. Su investigación suma un conjunto de análisis hechos por investigadores de todo el mundo, a partir de la misma grabación y transcripción de sesiones experimentales de diseño. En este caso la tarea consistía en diseñar un dispositivo para cargar y sujetar una backpack a una bicicleta de montaña. El momento del "salto creativo" ocurrió en torno al concepto de una "charola". En su estudio Cross identifica cinco estrategias que producen el salto: combinación (un panel+ una bolsa = charola); mutación (de un panel plano a una charola), analogía (de bolsa con charola), principios fundamentales (identificar requerimiento y funciones deseadas hasta llegar a una forma apropiada: charola) y emergencia (propiedades que no habían sido reconocidas y que subyacen en el diseño son identificados). Cross argumenta que más que un salto, lo que ocurre es que el concepto clave, en este caso "charola", funciona como un puente entre el espacio del problema y el espacio de solución. El factor crucial radica en unir los modelos parciales de sub-problemas y sub-soluciones a través de un concepto que permite que se extiendan uno sobre el otro. El puente encarna claramente la relación satisfactoria entre problema y solución. En palabras de Cross, "es el reconocimiento del concepto apropiado lo que se vive como una iluminación. "(Cross, 2006, p. 57). Es posible clarificar cómo se tienden estos puentes a través de citar un fragmento de la novela de J.M. Coetzee, The Master of Petersburg (1990). Aquí el autor encuentra "un puente" en el concepto de "Orfeo sin lira". El protagonista de la novela es Dostoievski, quien a lo largo de las primeras páginas ha intentado entrar en contacto con su hijo muerto sin lograrlo; ha explorado múltiples maneras de zanjar este problema sin hallar un camino, pero aquí ocurre algo mágico cuando se da una mutación en la figura de Orfeo. En el mito clásico Orfeo logra abrir las puertas del infierno para rescatar a su esposa gracias a la manera en que toca su lira. Veamos cómo Coetzee rediseña el mito:

8 Este puente no sólo se siente de manera implícita en los párrafos de la obra de J.M. Coetzee, sino que él mismo hace referencia a este concepto al principio de su novela Elizabeth Costello (2004). Ahí el puente aparece relacionado con el acto creativo y con los problemas cotidianos que todo mundo enfrenta: "THERE IS FIRST of all the problem of the opening, namely, how to get us from where we are, which is, as yet, nowhere, to the far bank. It is a simple bridging problem, a problem of knocking together a bridge. People solve such problems every day. They solve them, and having solved them push on." (Coetzee, 2004, p. 1.)
He is trying to cast a spell. But over whom, over a ghost or over himself? He thinks of Orpheus walking backwards step by step, whispering the dead woman's name, coaxing her out of the entrails of hell: of the wife in graveclothes with the blind, dead eyes following him, holding out limp hands before her like a sleepwalker. No flute, no lyre, just the word, the one word, over and over. When death cuts all other links, there remains still the name. Baptism: the union of the soul with the name, the name it will carry into eternity. Barely breathing, he forms the syllables again: Pavel (Coetzee, 1994, p. 5).

El párrafo empieza por hacer preguntas estúpidas, para después tratar de entender su situación a través de la analogía con Orfeo. El momento clave sucede cuando aparece la idea "Orfeo sin lira" que, si bien no se menciona de manera explícita, es la imagen que opera un cambio a la mitad del párrafo. A través de ella el protagonista encuentra una manera de explicarse cuál es su situación y lo que debería hacer; es decir, tiende un puente entre el espacio del problema y el espacio de solución. De la misma manera que el concepto de "charola" soluciona una serie de sub-problemas, aquí Orfeo sin lira cumple varias funciones. No sólo explica la terrible frustración de cualquier padre que busca a su hijo muerto, sino que es un concepto "apropiado" para caracterizar al protagonista, quien a diferencia de Orfeo, no es un poeta sino un novelista, es decir no tiene la música del verso, sino sólo la palabra. Pero este sencillo concepto logra aún más. A través de su imagen, el protagonista tiende un puente entre la tradición griega y la tradición cristiana. A través de restarle su arma, su lira, emerge la figura de Jesús, con su énfasis en la palabra y en la vida eterna. Nigel Cross utiliza el famoso dibujo de Jastrow para ilustrar la manera en que el reconocimiento del concepto apropiado encarna simultáneamente el problema y la solución. Bajo el esquema del pato que se convierte en el conejo, podemos entender el problema de Orfeo que se traduce en la solución de Jesús. En estos fenómenos de emergencia la idea de puente es central para dar cuenta del proceso creativo. ${ }^{8}$

Para concluir me gustaría abordar algunos aspectos de la elaboración de historias que se comprenden mejor a la luz de una de las prácticas fundamentales del diseño: hacer bocetos, modelos y prototipos. Bruce Mau la denomina "hacer la esperanza visible" y, junto con suscitar preguntas estúpidas y saltar bardas, constituye una de las actividades básicas y universales del diseño. A través de los bocetos, el diseñador dialoga con sí mismo. Al plasmar sus ideas pueden entenderlas, corregirlas y extenderlas. Para Tim Brown los bocetos y prototipos sirven para fallar temprano y repetidamente. Un pensamiento semejante ha sido planteado por Stephen Koch en la creación de historias. Los fracasos de las primeras versiones son grandes avances desde esta perspectiva, porque al mostrarnos lo que 
no funciona nos permiten enfocarnos en lo que funciona y continuar a partir de ahí con la exploración. Pero los bocetos también sirven en el diseño para moverse de manera simultánea y libre por diferentes planos de generalidad y de detalle. Es decir, permiten saltar de formulaciones generales sobre la solución y el problema, a formulaciones específicas de áreas más pequeñas. Esto encuentra su importante paralelismo en una rama de la literatura que es considerada a menudo sólo como una vertiente del cine: la escritura de guiones. Una de las analogías más frecuentes para explicar el lugar que ocupa el guión en el proceso de producción de una película es compararlo con los planos de un arquitecto. Sin embargo, hay una serie de paralelismos afines que no son explorados. Me refiero a la práctica de los guionistas de escribir sinopsis, argumentos y escaletas. La importancia y función de estas distintas prácticas se vuelve más elocuente si las pensamos como bocetos que abordan diferentes niveles de detalle. De igual manera que se recomienda al diseñador saltar continuamente entre la formulación general y la específica, permitiendo que cada una eche luces sobre la reformulación de la otra, lo mismo debería de recomendarse a los guionistas; es decir, saltar continuamente entre la sinopsis, argumento, escaleta y guión. Profundizar en esta veta de investigación podría arrojar interesantes resultado. Lo mismo puede decirse de los frutos que podrían obtener escritores y guionistas de las metodologías que el diseño ha desarrollado para el trabajo creativo colaborativo. Afortunadamente, durante las últimas décadas, los escritores de series de televisión han demostrado que la escritura creativa no es una prerrogativa del individuo y que se puede practicar en grupos. En este contexto, algunas recomendaciones del diseño podrían resultar relevantes para los escritores. Por ejemplo, las sugerencias de Cristopher Meinel y Larry Leifer de nunca cazar a solas; nunca regresar prematuramente a casa; cazar en pequeños equipos que maximicen la agilidad y la diversidad, escoger individuos con distintos talentos y provenientes de distintos contextos, alguno con la

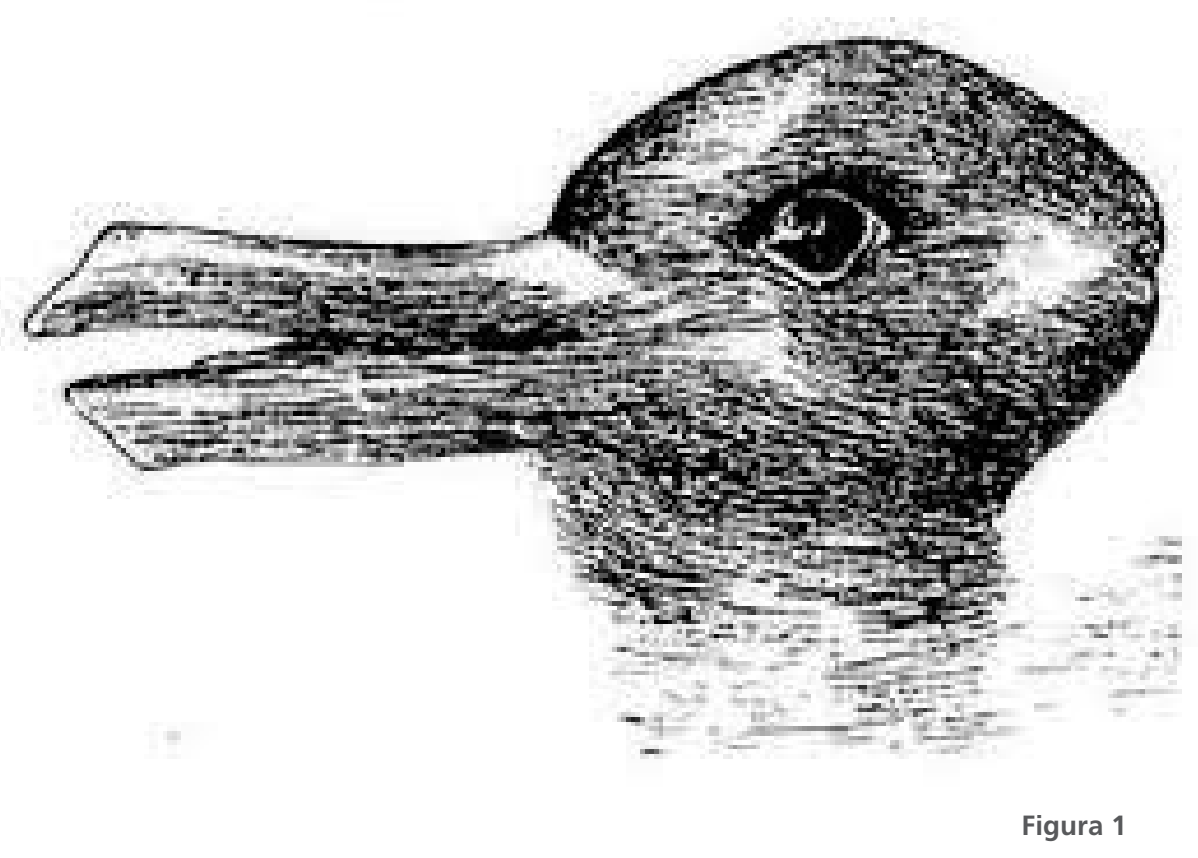

vocación instintiva del cazador, otro con el olfato optimizador del recolector, así como alguien encargado de la dinámica de grupo, de las emociones y de la comunicación (Meinel \& Leifer, 2012, p.1-2).

Con este bosquejo me gustaría terminar lo que ha sido una primera exploración de los múltiples niveles en que podría darse un diálogo entre la literatura y el diseño, dos disciplinas aparentemente lejanas, que, sin embargo, pueden iluminar sus prácticas, metodologías y debates. Este diálogo permite extraer nociones literarias de los desiertos de la especialización, abrir los sarcófagos, mostrar que los descubrimientos no sólo están en el futuro, en la tecnología y la ciencia, sino que pueden aparecer inesperadamente en el pasado, en la sabiduría y en la creatividad que durante siglos fue patrimonio de las letras. 


\section{Referencias}

Aristóteles. (1995). Poetics. Cambridge: Loeb Classical Library.

Auerbach, E. (2009). Philology and Weltliteratur The Princeton Sourcebook in comparative Literature. From the European Enlightenment to the Global Present (pp. 125.138). Prinstone: Princeton University Press.

Berger, W. (2010). CAD Monkeys, Dinosaur Babies and T- Shaped People. Inside the World of Design Thinking and How it Can Spark Creativity and Innovation. Nueva york: Penguin Books.

Bono, E. (1970). Lateral Thinking: A Textbook of Creativity. Londres: Ward Lock Educational.

Brooks, P. (1992). Reading for the Plot: Design and Intention in Narrative. Cambridge: Harvard University Press.

Brown, T. (2009). Change by Design: How Design Thinking Transforms Organizations and Inspires Innovation Harper Business. Nueva York HarperBusiness.

Buchanan, R. (1989). Declaration by Design: Rhetoric, Argument and Demonstration in Design Practice. In V. Margolin (Ed.), Design Discourse. History, Theory, Cristicism (p. 93). Chicago: University of Chicago Press.

(1995a). Rhetoric, Humanism and Design.

In R. Buchanan \& V. Margolin (Eds.), Discovering Design. Explorations in Design Studies (pp. 30-36). Chicago: University of Chicago Press.

(1995b). Wicked Problems in Design Thinking. In R. Buchanan \& V. Margolin (Eds.), The idea of Design. Cambridge: MIT Press.

Coetzee, J. M. (2004). Elizabeth Costello. Eight Lessons. Lodres: Vintage.

(1994). The Master of Petersburg. Londres:

Penguin Books.

Cross, N. (2006). Designerly Ways of Knowing.

Londres: Springer.

Dewey, J. (1960). The Quest of Certainty. A Study

of the Relation of Knowledge and Action.

Nueva York: Carpicorn Books.
Eagleton, T. (1996). Literary Theory. An introduction (2 ed.). Cambridge: Blackwell Publishing.

Edelman, J., Agarwal, A., Paterson, C., Mark, S., \& Leifer, L. (2012). Understanding Radial Breaks Design Thinking Research: Studying Co-Creation in Practice. Londres: Springer.

Gass, W. H. (2000). Reading Rilke: Reflections on the Problems of Translation. Nueva york: Alfred A. Knopf.

Hutcheon, L. (1988). A Poetics of Postmodernism: History, Theory, Fiction. Nueva York: Routledge.

Jakobson, R. (1995). Two Aspects of Language and Two Types of Aphasic Disturbances. Harvard: Harvard University Press.

Koch, S. (2003). Modern Library Writer's Workshop. A Guide to the Craft of Fiction. Nueva York: Random House.

Lindberg, T., Noweski, C., \& Meinel, C. (2010). Evolving discourses on design thinking: how design cognition inspires meta-disciplinary creative collaboration. Technoetic Arts: A Journal of Speculative Research, Vol. 8(No. 1), p. 32. doi: 10.1386/tear.8.1.31/1

Martin, R. (2009). Opposable Mind: Winning through Integrative Thinking. Harvard: Harvard University Business Press.

Meinel, C. (Larry Leifer). Understanding Radical Design Design Thinking Research: Studyng co-Creaction in Practice. Londres: Springer.

Rilke, R. M. (1991). Nuevos Poemas (F. Bermúdez-Cañete, Trans.). Barcelona: Hiperión.

Shklovsky, V. (1990). "Art as Device" Theory of Prose. Nueva York: Dalkey Archive Press.

Snow, C. P. (1964). The two cultures and a Second Look Nueva York: Mentor Books

Wang, J. (2013). The Importance of Aristotle to Design Thinking. Design Issues, 29 (2 primavera). 